\title{
Introduction
}

\section{Xavier Daumalin}

\section{OpenEdition}

\section{Journals}

Édition électronique

URL : https://journals.openedition.org/rhcf/1750

DOI : $10.4000 /$ rhcf. 1750

Éditeur

Rails \& histoire

Édition imprimée

Date de publication : 1 décembre 2003

Pagination : 22-26

ISBN : 0996-9403

ISSN : 0996-9403

\section{Référence électronique}

Xavier Daumalin, «Introduction », Revue d'histoire des chemins de fer [En ligne], 28-29 | 2003, mis en

ligne le 18 décembre 2014, consulté le 22 avril 2022. URL : http://journals.openedition.org/rhcf/1750 ; DOI : https://doi.org/10.4000/rhcf.1750 


\section{Xavier DAUMALIN}

\section{Introduction}

Les trois études suivantes ${ }^{1}$ évoquent l'histoire d'entreprises ferroviaires situées dans des zones géographiques relativement éloignées Rouen, Tours et Béziers - au cours des XIX et XX ${ }^{\mathrm{e}}$ siècles. Au-delà de la diversité des approches et des champs chronologiques retenus par les auteurs, ces recherches permettent de mettre en évidence trois thèmes : les différents modes de formation des ateliers de réparation du matériel ferroviaire ; les stratégies des ateliers face aux évolutions des marchés ; les problèmes liés à la gestion de l'espace.

\section{La formation des ateliers de réparation ferroviaire}

Si les modalités de création varient considérablement suivant la période retenue, il est néanmoins possible de dégager des constantes à l'intérieur même de chaque époque. Au début du XIx siècle, par exemple, lorsque les ateliers de réparation s'occupent également de la construction du matériel roulant, la plupart des entreprises françaises font ainsi largement appel aux grands spécialistes en la matière : les ingénieurs et les ouvriers anglais. Nous l'avions déjà constaté à La Ciotat avec le rôle éminent joué par George et Robert Stephenson entre 1839 et 1848, mais c'est encore plus net aux ateliers de Rouen étudiés par Michel Croguennec. En 1840, lorsque les banquiers Charles Laffitte et Edward Blount obtiennent la concession d'une ligne de chemin de fer reliant Paris à Rouen par la vallée de la Seine, ils s'adressent presque exclusivement à des techniciens anglais : la pose des voies est assurée par l'ingénieur Joseph Locke ; le percement des tunnels et la construction des ponts sont réalisés par les entrepreneurs Mackensie et Brassey. Quant aux opérations de construction et de réparation du matériel de traction - une quarantaine de locomotives environ -, elles sont confiées aux ingénieurs William Allcard et William Buddicom qui installent une usine à proximité du port de Rouen, au Petit-Quevilly.

Les études consacrées aux ateliers de Tours et de Béziers, fondés dans les années 1920, mettent en lumière une autre tendance, sans doute moins connue que la précédente. Au lendemain des grandes grèves de 1919-1920, certaines compagnies ferroviaires décident en effet de

1- Les communications de M. Balso, de M. Croguennec, de M. Monteil et de Mme Colombier ont été présentées par M. Daumalin dans cette synthèse qui a introduit le débat concluant la séance « Histoires d'ateliers, histoire d'entreprises ». Nous avons choisi de la publier en introduction au texte intégral des quatre auteurs (N.d.l.R.). 
sanctionner les cheminots grévistes en fermant leurs ateliers de maintenance et en sous-traitant les opérations de réparation à de nouvelles entreprises, spécialement créées pour l'occasion. A Tours, où 60 à $75 \%$ des cheminots ont participé à la grève générale de mai 1920, Bernard Monteil et Christine Colombier nous apprennent ainsi que la Compagnie du Paris-Orléans révoque plus de 2000 ouvriers et ferme ses ateliers de Saint-Pierre-des-Corps. Restée propriétaire des murs et des machines, la compagnie concède alors l'ensemble à une nouvelle société - la Compagnie générale de construction et d'entretien du matériel - pour une durée de quinze ans. Cette entreprise, dirigée par un ancien colonel, reçoit le renfort d'un cadre du Paris-Orléans et réembauche 700 des 2000 cheminots révoqués, en prenant bien soin d'exclure les militants syndicaux. La société complète ensuite ses effectifs avec des immigrés russes, tchèques et polonais. Toutes ces embauches se font bien entendu en dehors du statut du cheminot et se traduisent par une régression sociale : la journée de travail est portée de 8 à 9 h en 1923 et le travail du dimanche redevient courant. La communication de Raoul Balso dévoile un phénomène analogue à Béziers. Suite aux nombreuses grèves de 1919, la Compagnie du Midi licencie son personnel, ferme ses propres ateliers de réparation et sous-traite une partie de sa maintenance aux Établissements Fouga, une entreprise nouvellement créée dans laquelle figurent d'anciens ingénieurs de la Compagnie du Midi comme Henri Froustrey, Jacques Valette et Gaston Fouga. Les critères d'embauche du personnel ressemblent fort à ceux déjà évoqués à propos de la Compagnie générale de construction et d'entretien du matériel. Parmi les nouveaux venus, on retrouve d'anciens ouvriers de la Compagnie du Midi, vraisemblablement triés sur le volet, des métallurgistes venus du Nord et de l'Est de la France, des immigrés italiens, des travailleurs agricoles issus des exploitations viticoles voisines et quelques élèves de l'Ecole pratique de Béziers. Notons cependant, car toutes les études insistent sur ce point, qu'en dépit des précautions prises au moment de l'embauche ces ateliers issus de la répression sociale des années 1920 ne seront jamais des havres de paix sociale, bien au contraire. L'activité des Établissements Fouga sera constamment perturbée par des conflits plus ou moins graves entre la direction et le personnel, et ce mauvais climat social jouera un rôle non négligeable dans l'échec final de l'entreprise. Il en sera de même à la Compagnie générale de construction et d'entretien du matériel de Tours jusqu'à la réintégration de l'ancien personnel des ateliers de Saint-Pierre-des-Corps au sein de la SNCF. 


\section{Les stratégies des ateliers face aux marchés}

Ces trois communications abordent ensuite la question des différentes stratégies mises en œuvre par les ateliers ferroviaires face aux marchés. Il y a ceux, tout d'abord, qui sont spécialisés dans un petit nombre d'opérations destinées à des marchés bien spécifiques. C'est le cas, notamment, des Établissements Billard évoqués par Bernard Monteil et Christine Colombier. Après avoir débuté sa production industrielle en fabriquant des pièces automobiles, l'entreprise se lance en 1922 dans la construction ferroviaire en réalisant des draisines pour équiper les lignes départementales et les chemins de fer coloniaux comme le CongoOcéan. Quelques années plus tard, les Établissements Billard ajoutent à cette activité la production de locomoteurs et d'autorails, mais toujours pour les mêmes marchés.

D'autres ateliers ont au contraire toujours maintenu le même type d'activité depuis leur ouverture. L'exemple le plus révélateur est sans aucun doute celui des Ateliers de Quatre-Mares à Sotteville-lèsRouen. Ce site industriel, dont la création a été décidée par l'État en 1912 pour alléger la charge de travail et diminuer l'encombrement des vieux ateliers Buddicom, est à l'origine spécialisé dans la réparation des locomotives à vapeur pour l'ensemble de la région ouest. Vers la fin des années 1950, la traction Diesel commence à s'imposer et les Ateliers de Quatre-Mares décident alors de se préparer à cette évolution. Dès 1963, ils mettent en place un Service conversion Diesel spécialement chargé d'adapter les méthodes de travail de l'entreprise et, en 1965, les premières locomotives Diesel sortent des ateliers (la réparation des locomotives à vapeur sera définitivement arrêtée en 1968). Aujourd'hui encore, cet établissement demeure, avec celui de Nevers, le dernier établissement de la SNCF à assurer la maintenance des machines Diesel. Mais entre-temps, une autre adaptation s'est produite : depuis 1992, les Ateliers de Quatre-Mares ont en effet commencé à orienter une partie de leur activité vers la réparation des locomotives électriques. Grâce à cette stratégie d'adaptation systématique des méthodes de travail et des compétences aux différents temps forts du système de propulsion des locomotives, les Ateliers de Quatre-Mares - actuellement plus de 800 personnes - ont ainsi réussi à demeurer un des principaux sites de maintenance du matériel roulant de la SNCF.

On trouve enfin des ateliers qui, dès leur création, cherchent à diversifier leurs compétences et leurs marchés. Le meilleur exemple en la matière nous ramène à Béziers, aux Établissements Fouga. À ses débuts, l'entreprise ne s'occupe que de la réparation des wagons de 
marchandises de la Compagnie du Midi. Mais dans les mois qui suivent leur entrée en service, les Etablissements Fouga achètent de nouveaux terrains en bordure du canal du Midi et y construisent des ateliers destinés, cette fois, à la réparation des wagons-restaurants et des locomotives de la Compagnie du Midi. Dans les années 1930-1950 les opérations de diversification prennent un tour nettement plus spectaculaire. L'intégration de la Compagnie du Midi dans la toute nouvelle SNCF se traduisant par une baisse régulière des commandes de réparation, les Établissements Fouga se lancent dans de nouvelles productions : des boulangeries de campagne pour l'armée, des tracteurs pour les vignerons de la région, du matériel pour les forages pétroliers et les centrales thermiques, des pylônes de télécommunication, des citernes et, surtout, dans la construction d'avions. L'engagement des Établissements Fouga dans l'aéronautique débute en 1935 avec le rachat du site d'Aire-surAdour et c'est au début des années 1950 que cette aventure industrielle remporte un franc succès avec la sortie du fameux Fonga Magister. Cet avion sera rapidement commandé par l'armée française et par les armées de nombreux autres pays pour former et entraîner les pilotes de combat. Le département aviation des Établissements Fouga jouera un rôle déterminant dans la croissance de l'entreprise au lendemain de la Seconde Guerre mondiale.

\section{La gestion des espaces}

Les problèmes liés à la gestion des espaces se posent à toutes les entreprises et ne sont pas réservés, loin de là, aux seuls ateliers de réparation ferroviaire. Il semble cependant qu'en raison de la nature des opérations effectuées et des matériels employés, ils y jouent malgré tout un rôle plus important que dans bien d'autres branches. Ces différentes études révèlent que la question de la gestion des espaces se pose dès la fondation des sites industriels. Au début du XIX ${ }^{\mathrm{e}}$ siècle, il n'est en effet pas rare de constater des erreurs dans la localisation des premiers ateliers de réparation ferroviaire. Nous l'avions constaté avec l'exemple des ateliers Benet \& Cie à La Ciotat et c'est aussi le cas des Ateliers Buddicom, construits à proximité du port de Rouen. L'usine débute ses activités en 1842 et, quelques mois seulement après son entrée en service, le choix de son implantation se révèle déjà peu satisfaisant. Non seulement le site est trop étroit, sans possibilité d'extension, mais il n'est pas raccordé au réseau ferroviaire local. Les locomotives, dont le poids avoisine les vingt tonnes, doivent ainsi être acheminées par chariots hippomobiles jusqu'à la gare Saint-Sever, ce qui ne facilite pas la rapidité des opérations de maintenance, ni leur rentabilité. En décembre 1845, 
Allcard et Buddicom sont ainsi amenés à transférer leur usine du PetitQuevilly vers la commune de Sotteville-lès-Rouen, sur un terrain d'une douzaine d'hectares situé en bordure de la ligne de chemin de fer reliant Paris à Rouen.

La question de la gestion des espaces se pose ensuite au fur et à mesure de la croissance des établissements. Si au moment de leur fondation la plupart des ateliers sont construits dans des zones faiblement peuplées, l'urbanisation finit généralement par les rattraper et par bloquer leur possibilité d'extension. C'est ce qui se produit à Rouen dans les années 1920. Les Ateliers Buddicom étant devenus trop exigus, et l'entreprise étant désormais enclavée entre la ligne de chemin de fer et l'agglomération de Sotteville-lès-Rouen, un second site est créé par l'État non loin de là pour y transférer l'entretien des locomotives à vapeur (Ateliers de Quatre-Mares). Buddicom conserve les opérations de réparation des voitures ou des wagons et se retrouve ainsi en concurrence avec les ateliers de Rennes, du Mans et de Saintes. Bien mieux équipés que Buddicom pour ce genre de travaux, ces sites vont peu à peu entraîner la vieille entreprise anglaise sur la voie du déclin. La réorganisation des espaces industriels se traduit, dans ce cas, par une redistribution des métiers avec des conséquences économiques et sociales délicates à gérer.

La question de la gestion des espaces se pose enfin après la fermeture des ateliers de réparation. Que faire des établissements désaffectés et de la mémoire de ceux qui y ont consacré une grande partie de leur existence ? Quels bâtiments ou quelles machines conserver, avec quels financements et pour quelles destinations ? Ces questions patrimoniales se posent à Rouen, Tours, Béziers et bien entendu en Arles où nous sommes réunis aujourd'hui. Le débat est ouvert : souhaitons qu'en permettant d'exposer différents points de vue, ce colloque réussira à dégager des pistes fructueuses. 